PROCEEDINGS OF THE

AMERICAN MATHEMATICAL SOCIETY

Volume 128, Number 12, Pages 3463-3464

S 0002-9939(00)05450-2

Article electronically published on June 7, 2000

\title{
ON A THEOREM BY FARB AND MASUR
}

\author{
KOJI FUJIWARA
}

(Communicated by Ronald A. Fintushel)

\begin{abstract}
Farb and Masur showed that an irreducible lattice in a semisimple Lie group of rank at least two always has finite image by a homomorphism into the outer automorphism group of a closed, orientable surface group. We point out that their theorem extends to the outer automorphism groups of a certain class of torsion-free, freely indecomposable word-hyperbolic groups.
\end{abstract}

Let $\Sigma_{g}$ be the closed, orientable surface of genus $g$ and let $\operatorname{Mod}\left(\Sigma_{g}\right)$ denote the mapping class group of $\Sigma_{g}$. Farb and Masur proved the following in [FM].

Theorem 1 (Farb-Masur). Let $\Gamma$ be an irreducible lattice in a semisimple Lie group of rank at least two. Let $h: \Gamma \rightarrow \operatorname{Mod}\left(\Sigma_{g}\right)$ be a homomorphism. Then $\operatorname{Im}(h)$ is finite.

Their proof uses a deep work on the Poisson boundary of mapping class groups by Kaimanovich-Masur $\mathrm{KM}$.

Let $G$ be a torsion-free, freely indecomposable word-hyperbolic group [G]. By Sela, there is a graph of groups decomposition of $G$ such that the edge groups are isomorphic to $\mathbb{Z}$ and the vertex groups are in two families; one is a collection of the fundamental groups of punctured surfaces $\left\{S_{i}\right\}_{i}$, which are called CMQ subgroups, and the other is rigid in the sense that the outer automorphism groups are finite. This graph decomposition, which is called JSJ-decomposition of $G$, is unique in a certain sense; in particular the set $\left\{S_{i}\right\}_{i}$ is an invariant of $G$ (Theorem 1.7 of $[\underline{\mathrm{S}}]$ ). We say that $G$ is orientable in terms of JSJ-decomposition if all $S_{i}$ is orientable.

Let $G$ be the fundamental group of $\Sigma_{g}$. $G$ is word-hyperbolic if $2 \leq g$. It is also known that $\operatorname{Mod}\left(\Sigma_{g}\right)$ is isomorphic to $\operatorname{Out}(G)$ (see, e.g. [I] ). The JSJ-decomposition of $G$ is a trivial decomposition with one CMQ subgroup, which is $G$ itself. Thus $G$ is orientable in terms of JSJ-decomposition as well. Theorem 1 has an immediate extension as follows.

Corollary 2. Let $\Gamma$ be an irreducible lattice in a semisimple Lie group of rank at least two. Let $G$ be a torsion-free, freely indecomposable word-hyperbolic group. Suppose $G$ is orientable in terms of JSJ-decomposition. Let $h: \Gamma \rightarrow \operatorname{Out}(G)$ be a homomorphism. Then $\operatorname{Im}(h)$ is finite.

Received by the editors December 11, 1998 and, in revised form, February 8, 1999.

1991 Mathematics Subject Classification. Primary 20F32; Secondary 20F34, 22E40, 32G15.

Key words and phrases. Word-hyperbolic groups, JSJ-decomposition, lattices in higher-rank Lie groups, mapping class groups. 
Proof. Since the JSJ-decomposition is canonical in terms of $\operatorname{Out}(G) \operatorname{Out}(G)$ contains a subgroup of finite index, $A$, which is isomorphic to

$$
\mathbb{Z}^{n} \oplus\left(\bigoplus_{i=1}^{m} \operatorname{Mod}\left(S_{i}\right)\right),
$$

where $n$ is the number of edges in the JSJ-decomposition and $m$ is the number of CMQ subgroups (Theorem 1.9 of $[\mathrm{S}]$ ). Put $\Gamma^{\prime}=h^{-1}(A)$ and $h^{\prime}=\left.h\right|_{\Gamma^{\prime}}$. Since $\Gamma^{\prime}$ is of finite index in $\Gamma$, it suffices to show that $\operatorname{Im}\left(h^{\prime}\right)$ is finite. Clearly $\Gamma^{\prime}$ is a lattice. Let $p_{i}$ be the canonical projection from $A$ to $\operatorname{Mod}\left(S_{i}\right)$. By our assumption all $S_{i}$ are orientable. Apply Theorem 1 to $p_{i} \circ h^{\prime}: \Gamma^{\prime} \rightarrow \operatorname{Mod}\left(S_{i}\right)$ and obtain that $\operatorname{Im}\left(p_{i} \circ h^{\prime}\right)$ is finite for all $i$. Note that Theorem 1 holds for a compact, orientable surface with punctures as well (their proof includes this case, although the theorem may not clearly state it). Let $p$ be the projection from $A$ to $\mathbb{Z}^{n}$. To finish the proof it suffices to show that $\operatorname{Im}\left(p \circ h^{\prime}\right)$ is finite. Margulis' normal subgroup theorem says that a normal subgroup of a lattice in the class we are discussing is either finite or of finite index. Apply this theorem to $\operatorname{Ker}\left(p \circ h^{\prime}\right)$. It is impossible for $\operatorname{Ker}\left(p \circ h^{\prime}\right)$ to be finite. Therefore $\operatorname{Im}\left(h^{\prime}\right)$ is finite (indeed trivial).

The author suspects that Theorem 1 might hold for compact, but non-orientable surfaces $S$ as well. If so, one could remove the orientability assumption from Corollary 2 as well. But he could not find an argument for it. One might take a two sheeted covering $T$ of $S$ and try to reduce the problem to a certain subgroup of $\operatorname{Mod}(T)$ (see $[\mathrm{BH}])$. Another way would be to study the Poisson boundary of $\operatorname{Out}(G)$ for a word-hyperbolic group $G$, which is interesting for its own sake. The author would like to thank Nariya Kawazumi for useful informations. He is grateful to Benson Farb for explaining their work to him.

\section{REFERENCES}

[BH] J. Birman, H. Hilden, On isotopies of homeomorphisms of Riemann surfaces. Ann. of Math. (2) 97 (1973), 424-439. MR 48:4305

[FM] B. Farb, H. Masur, Superrigidity and mapping class groups, Topology. 37 (1998), No. 6, 1169-1176. MR 99f:57017

[G] M. Gromov, Hyperbolic groups. Essays in group theory, 75-263, MSRI Publ. 8, Springer, 1987. MR 89e:20070

[I] N. V. Ivanov, Algebraic properties of mapping class groups of surfaces. Geometric and algebraic topology, 15-35, Banach Center Publ. 18, PWN, Warsaw, 1986. MR 89a:57009

$[\mathrm{KM}]$ V. A. Kaimanovich, H. Masur, The Poisson boundary of the mapping class group. Invent. Math. 125 (1996), No. 2, 221-264. MR 97m:32033

[S] Z. Sela, Structure and rigidity in (Gromov) hyperbolic groups and discrete groups in rank 1 Lie groups. II. Geom. Funct. Anal. 7 (1997), No. 3, 561-593. MR 98j:20044

Mathematical Institute, Tohoku University, Sendai, 980-8578 Japan

E-mail address: fujiwara@math.tohoku.ac.jp 